Relations industrielles

Industrial Relations

\title{
Bernier, Jean, dir., Grèves et services essentiels/Strikes and
}

Essential Services

\section{Michel Grant}

Volume 51, numéro 1, 1996

URI : https://id.erudit.org/iderudit/051085ar

DOI : https://doi.org/10.7202/051085ar

Aller au sommaire du numéro

Éditeur(s)

Département des relations industrielles de l'Université Laval

ISSN

0034-379X (imprimé)

1703-8138 (numérique)

Découvrir la revue

Citer ce compte rendu

Grant, M. (1996). Compte rendu de [Bernier, Jean, dir., Grèves et services essentiels/Strikes and Essential Services]. Relations industrielles / Industrial Relations, 51(1), 215-218. https://doi.org/10.7202/051085ar

Tous droits réservés (C) Département des relations industrielles de l'Université Laval, 1996
Ce document est protégé par la loi sur le droit d'auteur. L'utilisation des services d'Érudit (y compris la reproduction) est assujettie à sa politique d'utilisation que vous pouvez consulter en ligne.

https://apropos.erudit.org/fr/usagers/politique-dutilisation/ 
variation in national culture and resources, and the variety in stages of industrialization. A second theoretical perspective discussed in the introduction and referred to in some of the nation-studies is the 'Dependency' perspective, in which industrialization is regarded as a consequence of foreign investment. The editors also reject this perspective because it attaches too much importance to multinationals as direct investors and underestimates the great variety in sources and composition of foreign direct investments.

Indeed, most of the chapters are illustrative of the great variation in national policies, industry development and company restructuring, and they show the mediation of foreign influence by the local context. However, in their rejection of the dependency perspective the editors seem to be at variance with some authors who stress this dependency, in the form of political or market compliance with demands put forward by foreign companies. That does not preclude national or industry variations in response to these demands, due to differences in the local or national context. A number of authors stress both the multinationals' influence and the specific industry of national response to it, rejecting the idea that this influence and dependence leads to international uniformity in labour rela- tions, but not denying the high degree of dependence.

One interesting contrast in this volume, in addition to the one between East Asia and South Africa, is between low wage countries doing everything they can to maintain this advantage, for instance by curbing the unions, and other countries, where the national government and enterprise management almost cooperate in their shift to a high wage economy, in which the labour movement plays a more active role. The difference is exemplified by the chapters on HRM in Korean manufacturing and on South African mesocorporatism, as opposed to some of the chapters in Part I.

Actually the only major point of criticism I can think of, is the lack of a concluding comparative survey. The introduction is a fine piece on 'the state of the art', summarizing the contributions, but a concluding discussion would do more justice to the theoretical notions discussed in the introduction and probably offer a better framework in which there is a place for both foreign dependence and for the great variety in responses, as well as for the many ways in which foreign influence is exercised.

HANS SLOMP
Katholieke Universiteit Nijmegen

\section{Grèves et services essentiels/Strikes and Essential Services}

sous la direction de Jean BERNIER, Sainte-Foy, Les Presses de l'Université de Laval et Conseil des services essentiels, 1994, 300 p., ISBN 2-7637-7389-3.

La publication de cet ouvrage fait suite à un colloque organisé en 1993 par le Conseil des services essentiels et par l'École nationale d'administration publique. Le but de cette rencontre était de tracer un bilan du rôle du Conseil des services essentiels pendant ses dix premières années d'existence, et de caractériser l'expérience québécoise en la comparant aux systèmes développés dans d'autres pays industriels. Les contributions des experts réunies dans ce volume illustrent la très grande diversité interna- tionale dans les modes de définition et de maintien des services essentiels lors de conflits de travail: Lennart Aspegren (Suède), Jean Bernier et Madeleine Lemieux (Québec), Donald D. Carter (Ontario et juridiction fédérale), Peter Feuille (États-Unis), Gillian Morris (Angleterre), Jean Pélissier (France) et Tiziano Treu (Italie). L'ouvrage propose également une réflexion du philosophe Lukas K. Sosoe sur la justification éthique du droit de grève. Jean Bernier présente dans un autre chapitre la problématique 
générale de la détermination et du maintien des services essentiels de même qu'une synthèse des contributions de ses collaborateurs. François Delorme trace les principales orientations qui se dégagent des discussions des participants aux ateliers dans ce colloque. L'ouvrage se termine par le texte de la conférence de clôture d'Alfred Pankert sur des éléments de prospective internationale à l'égard de la protection du droit de grève et du maintien des services essentiels.

Comme le souligne Jean Bernier, la recherche d'un équilibre entre le droit de grève et le droit à la santé et à la sécurité de la population donne lieu à des encadrements juridiques et institutionnels très diversifiês selon les trois dimensions suivantes: les fondements du droit de grève, la notion même des services essentiels et les mécanismes juridiques et institutionnels de leur détermination. En plus d'avoir à décrire et à analyser le type d'encadrement prévalant dans leur pays respectif, les auteurs tentent de qualifier l'attitude des parties à l'égard du régime, à dresser un bilan de son efficacitê et à examiner des solutions alternatives.

Les auteurs ne répondent pas à toutes les questions concernant les différents aspects du mode de détermination et de maintien des services essentiels, mais il se dégage toutefois de l'ensemble des textes un portrait suffisamment complet pour informer le lecteur au sujet des systèmes étrangers, et pour caractériser le régime québécois par rapport aux autres régimes.

Les différences internationales se manifestent dès l'examen des fondements du droit de grève. Les situations varient de pays où ce droit ne repose sur aucune assise constitutionnelle (v.g. Canada, ÉtatsUnis) mais parfois sur une assise législative (v.g. selon les états américains ou selon les provinces canadiennes), à d'autres pays (France, Italie, Suède) qui assurent à des degrés divers une protection constitutionnelle au droit de grève tout en reconnaissant à l'État ou aux parties le pouvoir de réglementer ou d'encadrer son exercice. En Angleterre, même si les fondements du droit de grève ne se retrouvent pas dans une constitution ou dans un texte législatif, il ne s'en suit pas nécessairement que la loi anglaise empêche les salariés de déclencher un arrêt de travail dans les services essentiels.

Chaque pays ou chaque juridiction désigne à sa façon les services que sa population devra continuer à recevoir pendant une grève et définit d'une façon étroite ou large la notion de services essentiels. Peter Feuille suggère que la réalisation d'un consensus autour de la définition du caractère essentiel d'une activité dépend du seuil de tolérance d'une population à l'égard des inconvénients, de l'anxiété et des privations suscités par un conflit de travail (p. 124). Un État peut ainsi considérer un service essentiel dans sa globalité et prohiber le droit de grève tout en instaurant des mécanismes alternatifs d'arbitrage (v.g. services hospitaliers en Ontario; policiers au Québec; fonction publique fédérale aux États-Unis). La notion des services essentiels peut dans certains cas se limiter à la protection de la santé et la sécurité du public (v.g. services publics au Québec); elle peut s'exprimer par des pourcentages du personnel à maintenir en poste en cas de grève (v.g. établissements de santé au Québec), ou par des activités liées à des besoins sanctionnés par la constitution d'un pays tels la vie, la santé, la sécurité, la liberté, la sécurité sociale, l'éducation et la libre communication (v.g. Italie). Les personnes occupant certaines fonctions peuvent être désignées comme devant travailler pendant une grève (v.g. fonction publique fédérale au Canada). En France, la notion de services essentiels n'est aucunement utilisée, par contre le cadre juridique reconnait le droit de grève aux fonctionnaires tout en exigeant qu'il soit exercé en conformité avec la règle de la continuité du service public; cette 
dernière notion déborde largement le domaine de la santé et de la sécurité publique, et est cependant entendue de façon plus stricte dans certains secteurs que dans d'autres (v.g. enseignement, musées nationaux, hôpitaux et services de police). Comme le résume correctement Jean Bernier: "Il s'agit en fait d'une notion assez élastique, à géométrie variable, dont le contenu varie en fonction de divers facteurs politiques, historiques et culturels" (p. 58).

Bernier (p. 60-62) propose une typologie utile au lecteur qui veut se retrouver à travers les méandres des modes complexes et diversifiés de détermination des services essentiels. Il note d'abord un ensemble de règles communes à tous les États relatives: (1) à l'exclusion du droit de grève pour certains groupes (policiers, pompiers, armée, gardiens de prison), (2) à l'instauration d'un régime particulier dans les services publics, (3) au recours à des mesures applicables en cas d'urgence nationale ou de mouvements de grève incontrôlés (v.g. réquisition du personnel, lois spéciales, recours à l'armée), et (4) à la répression des grèves illégales (v.g. utilisation du personnel de remplacement, mesures disciplinaires, amendes aux syndicats et aux grévistes). Ensuite, Bernier (p. 62-68) propose trois modèles de détermination des services essentiels: d'abord celui de la voie légale ou réglementaire, ensuite celui de la voie volontariste ou consensuelle, et finalement celui de la voie mixte comprenant l'intervention d'un tiers. Ainsi, le régime s'appliquant dans la fonction publique américaine et dans la plupart des états américains s'inscrit dans la voie où par législation ou autrement, l'autorité gouvernementale assure le maintien de services essentiels en prohibant la grève; dans les établissements de santé au Québec, l'Assemblée nationale a réduit la grève à une manifestation symbolique, et ce sans prévoir des mesures alternatives pour le règlement des différends. La voie consensuelle laisse aux acteurs sociaux le soin de déterminer les services essentiels avec un minimum d'intervention de l'État. La Suède constitue sans doute la manifestation la plus " pure " du modèle volontariste puisque, comme le souligne Lennart Aspegren, les mesures spécifiques de protection de l'intérêt public à l'occasion d'un conflit de travail se retrouvent non pas dans des dispositions législatives, mais dans des accords nationaux s'appliquant tant dans le secteur public que dans le secteur privé (p. 248). Finalement, la voie mixte laisse aux parties, et en particulier aux syndicats, le soin de déterminer les services essentiels à maintenir; en cas de désaccord, un tiers peut intervenir. Ainsi en Italie, la pluralité de sources réglementant l'exercice du droit de grève dans les services essentiels reflète un choix politique favorisant l'approche consensuelle et reposant plus sur des règles procédurales que sur des règles substantives (p. 176-179).

Par rapport aux attitudes des parties, Donald D. Carter souligne l'ambivalence des organisations syndicales à l'égard d'un système qui prohibe la grève dans des secteurs importants, mais qui offre l'arbitrage comme mode de règlement final des différends et qui a souvent ainsi permis de réaliser des gains significatifs. La diminution du nombre de grèves dans les services essentiels atténue selon plusieurs l'urgence d'un débat sur cette question. Alfred Pankert conclut que le problème des services essentiels ne constitue plus un problème prioritaire dans la plupart des pays. Les questions reliées au chômage, au coût de la sécurité sociale et aux problèmes budgétaires des gouvernements représentent maintenant des préoccupations plus importantes pour les acteurs sociaux.

La synthèse des ateliers préparée par François Delorme confirme la crédibilité et l'expertise que le Conseil des services essentiels a développées au cours de ses interventions dans le secteur des services publics. Si le Conseil a réussi à préserver l'équilibre entre le droit de grève et la protection de la santé et la sécurité 
du public dans ce dernier secteur, cette tâche s'avère beaucoup plus problématique dans les établissements de santé ; la réduction de la grève à une dimension symbolique écarte la voie consensuelle et réduit significativement les possibilités d'une intervention crédible et efficace du Conseil.

Lukas K. Sosoe examine le droit de grève dans les services essentiels comme un défi sur le plan éthique. Il souligne combien la réflexion de la philosophie est peu avancée sur cette question. Sa thèse fondamentale propose que le droit de grève ne se justifie pas comme un bien en soi, mais comme un moyen subsidiaire seulement. Il suggère d'abord une réflexion sur le sens du travail: le travail comme source de dignité et de valorisation intrinsèques, le travail comme produit et comme créateur de richesses. Une telle valorisation du travail justifie-telle le droit de grève? Le travail peut humaniser, mais il peut aussi déshumaniser; la devise d'Auschwitz n'était-elle pas "Le travail libère! " ? Le travail est aussi un lieu où se développe le conflit, et le droit de grève est un moyen de régulation pour corriger l'asymétrie qui favorise l'employeur aux dépens du salarié. Or le droit de grève est un droit exercé collectivement dont les effets ne restent pas localisés et affectent des tiers. Donc accepter le droit de grève implique que les droits de la collectivité vont être affectés. L'histoire du syndicalisme démontre que la grève apparaît comme un moyen de lutte et qu'en tant que moyen, il est substituable et sa nécessité se trouve relativisée. Sosoe souligne qu'on peut concevoir d'autres moyens de lutte et de négociation que la grève, et qu'en conséquence le droit de grève n'est pas un droit fondamental. Même si cette conclusion ne fait sans doute pas l'unanimité dans le milieu des relations du travail, ce texte a le mérite d'être le seul dans cet ouvrage à aborder la dimension éthique du droit de grève puisqu'elle n'est que brièvement évoquée par quelques uns des collaborateurs.

Ce volume répond à plusieurs types de besoin. D'abord, il réunit un ensemble de textes qui décrivent d'une façon synthétique et dans un style accessible divers régimes de détermination et de maintien des services essentiels. En plus de nous informer sur les régimes étrangers, cet ouvrage nous permet de constater combien la voie québécoise présente des attributs originaux avec une institution comme le Conseil des services essentiels, et qu'elle comporte des contrastes dans son approche à l'égard des services essentiels ; en effet, si le Québec définit d'une façon étroite les services essentiels à maintenir dans les services publics et y permet un recours efficace à la grève, les syndicats dans les établissements de santé n'ont accès ni à un droit grève effectif ni à l'arbitrage des différends.

Cinq chapitres sont écrits en anglais et six en français, mais tous sont suivis d'un résumé dans l'autre langue. La très grande diversité des modes de détermination et de maintien des services essentiels et le caractère collectif de l'ouvrage expliquent sans doute un certain manque d'uniformité dans la structure et l'organisation des chapitres écrits par les experts étrangers. Cette lacune n'affecte toutefois pas d'une façon significative la valeur des textes. Les praticiens, les chercheurs, les étudiants et tous ceux qui s'intéressent à cet aspect fondamental du régime québécois de relations du travail trouveront dans ce volume un outil précieux et une référence essentielle.

MICHEL GRANT

Université du Québec à Montréal 